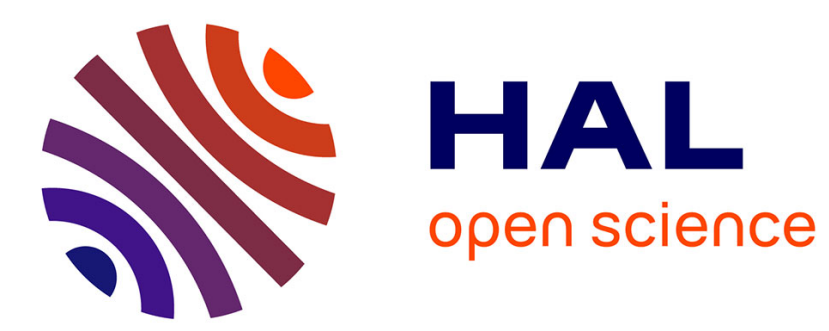

\title{
Territoires et paysages antiques du littoral septentrional de la mer Noire: nouvelles recherches à Taman (Russie) \\ Murielle Faudot
}

\section{To cite this version:}

Murielle Faudot. Territoires et paysages antiques du littoral septentrional de la mer Noire: nouvelles recherches à Taman (Russie). Dialogues d'histoire ancienne, 1996, 22 (2), pp.257-259. 10.3406/dha.1996.2724 . hal-02964721

\section{HAL Id: hal-02964721 \\ https://hal.science/hal-02964721}

Submitted on 12 Oct 2020

HAL is a multi-disciplinary open access archive for the deposit and dissemination of scientific research documents, whether they are published or not. The documents may come from teaching and research institutions in France or abroad, or from public or private research centers.
L'archive ouverte pluridisciplinaire $\mathbf{H A L}$, est destinée au dépôt et à la diffusion de documents scientifiques de niveau recherche, publiés ou non, émanant des établissements d'enseignement et de recherche français ou étrangers, des laboratoires publics ou privés. 
Territoires et paysages antiques du littoral septentrional de la mer Noire : nouvelles recherches à Taman (Russie)

Madame Murielle Faudot, Monique Clavel-Lévêque

\section{Citer ce document / Cite this document :}

Faudot Murielle, Clavel-Lévêque Monique. Territoires et paysages antiques du littoral septentrional de la mer Noire : nouvelles recherches à Taman (Russie) . In: Dialogues d'histoire ancienne, vol. 22, n², 1996. pp. 257-259;

doi : https://doi.org/10.3406/dha.1996.2724

https://www.persee.fr/doc/dha_0755-7256_1996_num_22_2_2724

Fichier pdf généré le 16/05/2018 


\section{Territoires et paysages antiques du littoral septentrional de la mer Noire : nouvelles recherches à Taman (Russie)}

Corollaire, entre autres, des contacts entre Grecs et indigènes, la question des territoires coloniaux et des paysages antiques $\mathrm{du}$ littoral nord-est de la Mer Noire échappe encore aux démarches de synthèse. Ainsi cette forme des relations n'a-t-elle été abordée que de loin en loin lors du $6^{\mathbf{e}}$ symposium de Vani1 ${ }^{1}$, tandis que la résonance des travaux sur Chersonèse Taurique et Olbia Pontique masquait les résultats encourageants des recherches menées dans la région de Taman. C'est quelques informations concernant cette dernière région que nous voudrions présenter aujourd'hui.

Située à l'Est du Détroit de Kertch, entre la mer d'Azov et la mer Noire, la péninsule de Taman est constituée de la presqu'île de Fontalovski, au Nord, et de la presqu'île de Taman, au Sud ; cet espace a été colonisé par les Grecs dans la première moitié du $\mathrm{VI}^{\mathrm{e}}$ siècle avant notre ère, lors de la deuxième vague de colonisation de cette partie du Pont-Euxin2.

On dispose de données archéologiques provenant des cités ioniennes de Patrasys, Képoï, Phanagoria et Hermonnassa et, depuis une date récente, d'indications sur l'occupation de leur territoire ainsi que sur le paysage antique.

Ces dernières informations résultent d'un programme de recherche développé depuis 1992 par l'Institut d'Archéologie de Moscou (Académie des Sciences de Russie) qui porte particulièrement sur l'organisation du territoire agricole et sur les systèmes d'utilisation de la terre dans la partie asiatique de l'État du Bosphore. Un premier bilan de ces recherches, dressé par Youri Gorlov et Youri Lopanov, rend compte des données provenant essentiellement de prospections de terrain et de la photointerprétation ${ }^{3}$.

Sur l'ensemble de la péninsule apparaissent des réseaux de lignes et des réticulés de parcelles, qui consistent sur le terrain en

1. LÉVÊQUE (P.), LORDKIPANIDZE (O.) dir., Sur les traces des Argonautes. Actes du 6e symposium de Vani (Colchide), 22-29 septembre 1990, Besançon, 1996.

2. Cette périodisation est proposée dans : KOCHÉLENKO (G.), KOUZNETSOV (V.), La colonisation grecque du Bosphore cimmérien, in Le Pont-Euxin ou par les Grecs, symposium de Vani (Colchide), septembre-octobre 1987, Lévêque (P.), Lordkipanidze (O.) dir., Besançon, 1990, p. 67-84.

3. GORLOV (Y.), LOPANOV (Y.), Système antique de bonification des sols sur la presqu'île de Taman, VDI, 1995-3, p. 121-137 (en russe). 
levées de terre dont la hauteur conservée atteint parfois le mètre pour une largeur qui peut aller jusqu'à 20 mètres. La répartition des bourrelets artificiels a été cartographiée sur la presqu'île de Fontalovski ${ }^{4}$ : une vingtaine d'ensembles, souvent discontinus, a été reconnue. La surface de chacun de ces réseaux est comprise entre 1,5 et $2 \mathbf{k m}^{2}$, sauf pour la partie nord-ouest de la presqu'île qui est couverte par un maillage dense de $12,5 \mathrm{~km}^{2}$. Ces structures, déjà inventoriées par Iakov Paromov5, sont interprétées par les auteurs de l'article comme étant des éléments d'un système antique de bonification des sols, destinés à la régularisation de l'écoulement des eaux de pluie. On note avec intérêt l'observation que les surfaces des parcelles dessinées par ces levées de terre sont d'autant plus importantes que la pente du terrain est faible et inversement.

Outre les conditions historiques, un réchauffement climatique manifeste entre les IV $^{\mathbf{e}}$ et $\mathrm{I}^{\mathrm{er}}$ siècles avant notre ère, expliquerait cette forme de mise en valeur des sols. De fait la datation proposée de ces structures est comprise dans la même fourchette chronologique, entre les IVe et $I^{\text {er }}$ siècles avant notre ère. Ces parcellaires seraient donc postérieurs aux quatre phases de la colonisation grecque du Bosphore cimmérien, telles que Guénadi Kochélenko et Vladimir Kouznetsov les ont définies 6 .

Principalement focalisé sur la région de Fontalovski, ce premier volet de l'étude est désormais prolongé par un programme de recherches franco-russe sur les paysages antiques et le territoire d'Hermonassa, devenu celui de Taman.

Lors de la phase initiale de ce travail, qui s'est déroulée à Moscou à la fin de 1996, les équipes moscovite et bisontine ont analysé un échantillon d'un peu plus de 1400 photographies aériennes?.

Au terme de l'étude préliminaire, des résultats apparaissent qui confirment la prégnance de l'information au sol et la rare qualité des clichés russes. L'existence sur la presqu'île de Taman de

4. GORLOV (Y.), LOPANOV (Y.), ibid., p. 123 et 136.

5. PAROMOV (I.), Intervention sur la presqu'île de Taman, in Lévêque (P.), Lordkipanidze (O.) dir., Le Pont-Euxin ..., op. cit. p. 161-164. La note 1 donne les références antérieures à ces recherches.

6. KOCHÉLENKO (G.), KOUZNETSOV (V.), loc. cit., p. 81-82.

7. Une convention de coopération a été signée entre l'Institut d'Archéologie de Moscou et l'Administration de la région de Krasnodar d'une part, et les équipes françaises universitaires et CNRS de la Maison de l'Orient (Lyon), les GDR 926 et URA 338 (Besançon), le GDR 1056 (Aix-en-Provence) et l'École Française d'Athènes d'autre part. 
structures agraires du type de celles connues sur la presqu'île de Fontalovski est vérifiable sur de nombreux documents. On reconnaît sur les photographies un marquage identique à celui observé à Fontalovski : des réseaux de lignes sombres qui se coupent à angle droit, ou proche de l'angle droit, délimitent des alvéoles, des parcelles. Sur certains clichés, de grands arcs de cercles sombres constituent ce qui pourrait être comparé à de grands enclos de pacage. Le parcellaire non orthogonal qui leur est associé est de dimensions supérieures au précédent.

Les groupes de traces, tous types confondus, sont essentiellement localisés dans la partie Sud-Ouest de la presqu'île, à faible distance des côtes. On note également leur présence à proximité immédiate de la ville de Taman (au Sud-Ouest), et au centre de la presqu'île, à Progress, Vinogradni et Vuchesteblieskaia. L'antiquité des traces reste à garantir pour chacune de ces zones, c'est pourquoi une prospection des ensembles de traces est indispensable, afin de vérifier au sol la nature du marquage et d'effectuer une confrontation avec la carte archéologique.

Juxtaposés à ces éléments on trouve fréquemment des kourganes. Ces vestiges soulèvent par leur présence le problème de l'organisation du territoire d'Hermonassa entre population scythe et grecque. Cette question reste largement à explorer avec, à long terme, l'objectif d'arriver à établir un phasage des transformations formelles et fonctionnelles de l'espace.

Parallèlement à cette périodisation des formes et de la nature de l'occupation de l'espace, les informations hydrographiques, géologiques et pédologiques, nécessaires à l'approche du paléoenvironnement et de l'évolution des paysages depuis l'antiquité jusqu'à l'époque contemporaine, sont actuellement en cours d'analyse à partir des données de l'imagerie satellitaire (SPOT panchromatique et multispectrale).

Murielle FAUDOT

ISTA, CNRS - Université de Franche-Comté 